\title{
RETROVESICAL HYDATIDOSIS ASSOCIATED WITH URINARY TRACT PATHOLOGY - CASE REPORT
}

\author{
ENIKÖ BARABÁS-HAJDU ${ }^{1}$, ADRIAN MAIER ${ }^{2 *}$, \\ FLORIN COROŞ ${ }^{3}$ and ORSOLYA MÁRTHA ${ }^{2}$ \\ ${ }^{1}$ Department of Microbiology, Pharmacology and Clinical Pharmacy, \\ University of Medicine and Pharmacy, Targu-Mures, Romania \\ ${ }^{2}$ Department of Urology, University of Medicine and Pharmacy, Targu-Mures, Romania \\ ${ }^{3}$ Department of General Surgery, University of Medicine and Pharmacy, \\ Targu-Mures, Romania
}

(Received: 31 July 2014; accepted: 30 October 2014)

Cystic hydatidosis $(\mathrm{CH})$ is a worldwide distributed parasitic zoonosis. It is considered one of the 17 neglected parasitic tropical diseases, among cysticercosis and soil transmitted helminthiases. $\mathrm{CH}$ is caused by the larval stage of Echinococcus granulosus, a tapeworm that usually infects dogs and other carnivorous animals as definitive hosts and herbivorous animals and rarely humans as intermediate hosts. Main primary localizations are the liver and the lung. In less than $3 \%$ they can primarily be present in the spleen. Treatment is mainly surgical, in some cases resulting in reoccurrence. In this paper we present the case of a male 55 years old patient who underwent a surgical intervention on his spleen for a solitary hydatid cyst as primary localization. Fifteen years after the operation the patient presented macroscopic haematuria; routine laboratory findings presented soft eosinophilia, 5\%, without any other modification. There was found no palpable tumour in the pelvis by rectal examination. Abdominal ultrasound investigation revealed a $2 \times 1 \mathrm{~cm}$ formation in the urinary bladder at the base of the left bladder-wall and a retrovesical, inhomogeneous $10 \times 10 \mathrm{~cm}$ tumour with multiple septa and transonic zones. Computed tomography (CT) scan strongly suggested the presence of a bladder tumour and a hydatid cyst. The symptoms caused by the bladder tumour revealed the co-existing nonsymptomatic retrovesical secondary $\mathrm{CH}$, which is a rare complication of splenic Echinococcus granulosus infection. Close follow-up and a proper pre- and postoperative anti-parasitic medication of the patient could have prevented reoccurrence of $\mathrm{CH}$.

Keywords: primary splenic hydatidosis, secondary retrovesical hydatidosis, bladder tumour

* Corresponding author; E-mail: maier23adrian@yahoo.com 


\section{Introduction}

Cystic hydatidosis $(\mathrm{CH})$ is a well-known parasitic disease described from ancient times. The proof that Echinococcus granulosus causes $\mathrm{CH}$ was brought by Carl von Siebold. In the year 1853 he reproduced the life cycle of the tapeworm by feeding cysts removed from sheep to dogs developing adult cestodes in their intestinal tract. The experiment was reproduced by Naunyn in 1863, using cysts from humans and feeding them to dogs with the same results [1]. Usually $\mathrm{CH}$ is well-tolerated by the host, depending on localization and diameter. Cysts grow $1-2 \mathrm{~cm}$ in diameter each year without having a symptomatic character. Main localizations of $\mathrm{CH}$ are the liver (60-70\%) and the lungs $(30 \%)$, only $10 \%$ of the cases passing these two filters. Primary splenic $\mathrm{CH}$ is very rare, less than $3 \%$ of the patients, being resolved usually by classical surgery without any recurrence. When complications appear, they manifest as secondary infections, compression of other organs, rupture in the abdominal cavity causing life-threatening generalized anaphylactic reaction or fistulization to other neighbouring organs, especially to the large bowel. For the postoperative prevention four week albendazole treatment and a six-month CT-scan follow-up should be respected [2].

Bladder neoplasms $(\mathrm{BN})$ are considered to be the most frequent cancers of the urinary tract [3]. They are the $7^{\text {th }}$ most common malignant tumours in men and the $17^{\text {th }}$ most common neoplasms in women. BN occur 3 to 4 times more frequent in men and have a higher incidence in developed countries of Europe and the USA [4]. A large percent, 70\%, of BN reside in the urothelium, causing non-muscle invasive bladder cancer [5]. The most influencing risk factors for developing BN are smoking and occupation, while diet and environmental conditions are less important in its aetiology [6].

The co-existence of bladder neoplasm and secondary retrovesical hydatid cyst is very rare in the literature and demands an interdisciplinary professional team to handle it.

\section{Case report}

A 55-year-old male patient presented with intermittent episodes of macroscopic haematuria at the urology ward. The patient had no other complaints. Physical examination revealed a scar in the left hypochondrium, resulting after a surgical intervention, which was performed 15 years ago for a solitary hydatid cyst of the spleen. There were no physical or laboratory modifications except a soft eosinophilia (5\%) and a macroscopic haematuria; no tumour mass was palpable on rectal examination. Chest radiography described no abnormalities. 


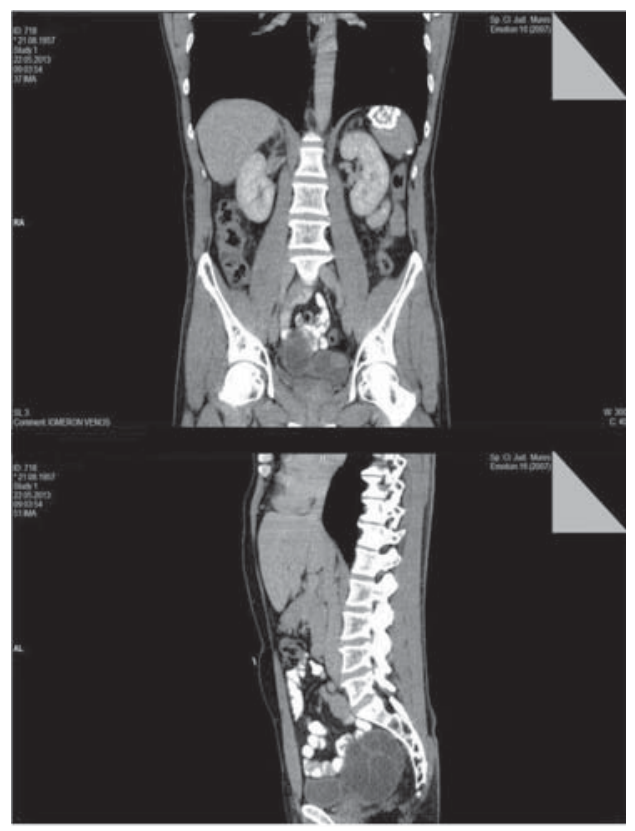

Figure 1. CT-scan showing a bladder tumour, retrovesical hydatid cyst, and a calcified scar in the spleen

Abdominal ultrasound examination revealed a $2 \times 1 \mathrm{~cm}$ sized tumour localized at the base of the left bladder-wall and a $10 \times 10 \mathrm{~cm}$ sized retrovesical inhomogeneous cyst with multiple septa and transonic zones suggesting a $\mathrm{CH}$. The abdominal CT-scan strongly suggested the bladder tumour and hydatid cyst diagnosis; it also revealed a spleen scar as a result of a surgical intervention for a primary $\mathrm{CH}$ (Fig. 1). The liver and the other abdominal organs were normal.

The strong CT suspicion of a retrovesical hydatid cyst indicated an oral treatment with a daily $400 \mathrm{mg}$ dose of albendazole for four weeks. Because of the existence of these comorbidities, a $\mathrm{CH}$ and a $\mathrm{BN}$, a team constituted of urologists and general surgeons resolved the case together.

The main question was: which pathology must be treated first? Because of the risk of haematuria, secondary the presence of bladder mass and the possible neoplastic character we performed first, a transurethral resection of the bladder tumour. After four weeks with an albendazole prophylaxis, the ablation of the hydatid cyst was executed. The presence of an important number of daughter vesicles, the risk of important haemorrhage (because of the localization of the cyst), with secondary anaemia and other possible comorbidities, an open surgery technique was indicated (Figs 2 and 3). 


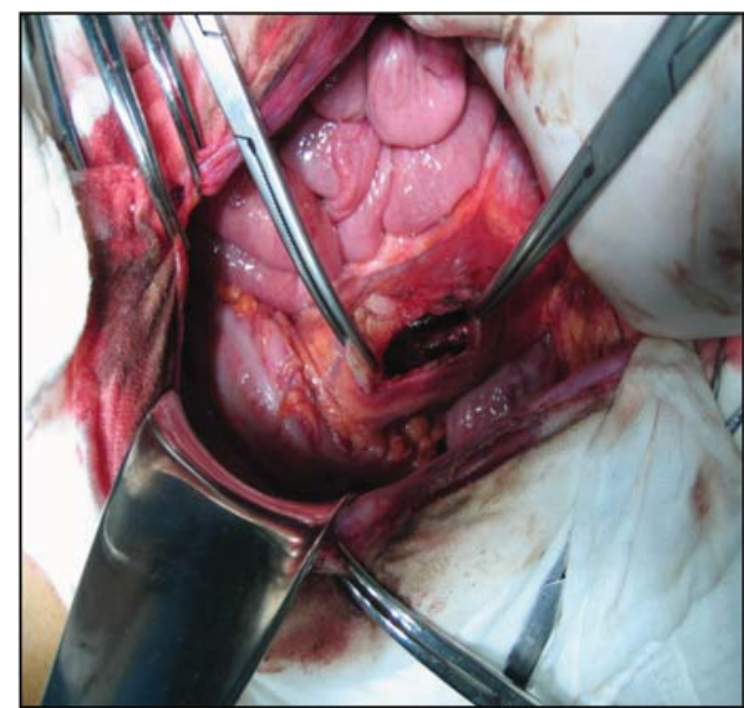

Figure 2. Intraoperatory findings during open surgery

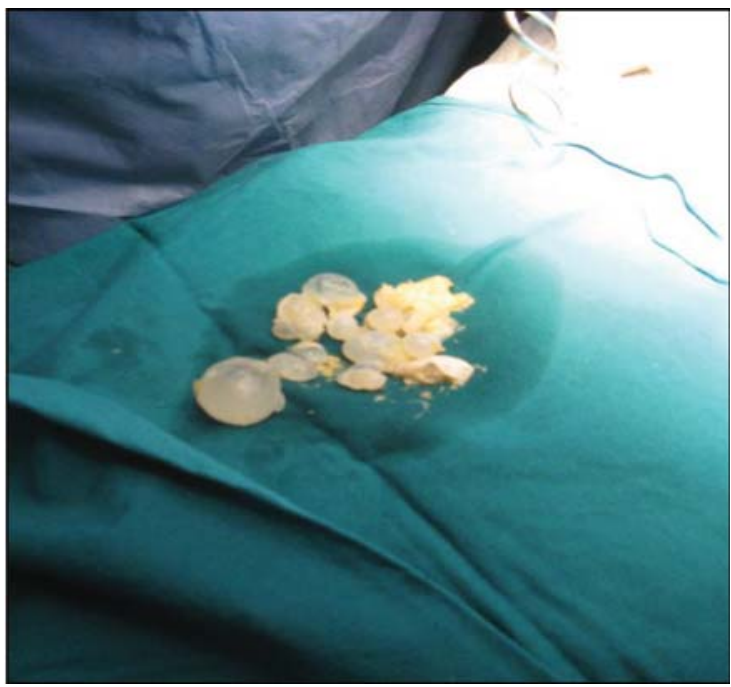

Figure 3. Hydatid vesicles removed from the patient

The removed vesicles underwent parasitological and histological examinations, which confirmed the diagnosis of hydatidosis. Histopathological examination of the BN revealed a G2 (tumour grading), low grade malignancy and a pTa (non-invasive papillary carcinoma) stadium tumour. There were no complica- 


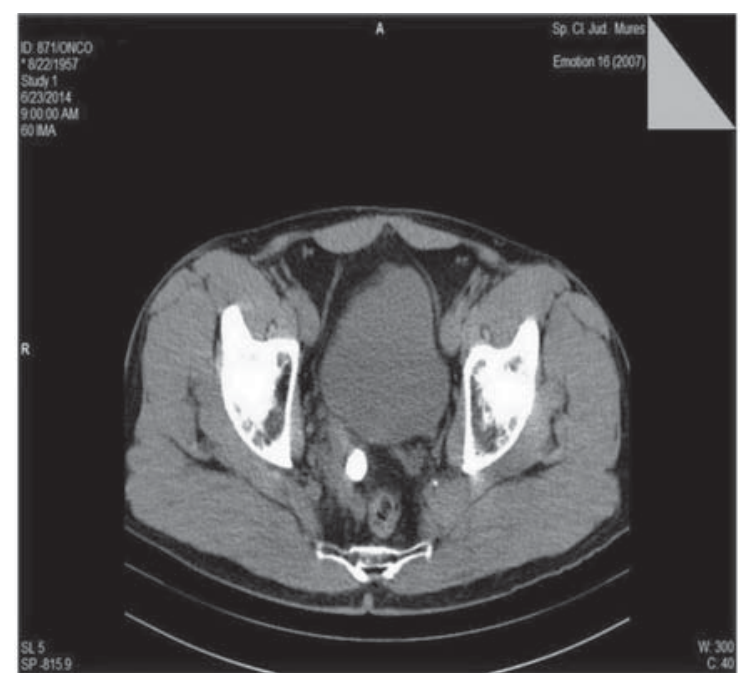

Figure 4. CT scan after one year, revealed normal bladder with small scar behind

tions after the operation and the patient was discharged on the seventh postoperatory day with the recommendation to continue the oral treatment with albendazole for another three weeks. For additional treatment of BN instillations with BCG were performed, based upon Lahm scheme.

After a six-week period the patient was examined again. CT scan revealed no changes in his condition. The postoperative control after 3 months showed no evidence of tumour recurrence and no re-occurence of hydatid cyst in the pelvic area. Control after one year showed no bladder tumour or hydatid recurrence (Fig. 4). The follow-up should continue for at least 3 years.

\section{Discussions}

Cystic hydatidosis $(\mathrm{CH})$ is a worldwide distributed parasitic zoonosis caused by the larval stage, metacestode, of Echinococcus granulosus. This parasite affects sheep, cattle and humans as intermediate hosts and dogs, wolves, foxes as definitive hosts. It is considered one of the 17 neglected parasitic tropical diseases; among cysticercosis and soil transmitted helminthiases [9].

Urinary tract localization of hydatid cysts is unusual representing only $2-4 \%$ of the cases [10]. Primary or secondary isolated hydatid cyst with retrovesical localization is also rare [11]. Diagnosis usually is based on physical examination revealing a non-symptomatic mass in the pelvis, completed by laboratory 
findings, such as eosinophilia and specific serological tests. X-ray, ultrasound, CT and MRI can also help to make the diagnosis, showing eggshell-like calcifications, multiple septa, daughter vesicles in the suspected area which can be represented by any organ or tissue of the human body [2].

The major concern in the management of pelvic $\mathrm{CH}$ is complication due to spillage of the highly immunogenic content that may lead to anaphylactic reactions or dissemination by implementation of the spilled scolices, Palanivelu hydatid system is a special instrument able to aspirate the cyst without spilling its content [12]. If necessary, open surgery is the best choice.

For the management of the $\mathrm{BN}$, a transurethral tumour-removal is recommended. The extracted materials undergo histopathological examinations. In case of $\mathrm{BN}$, they help to define the malignancy grade and guide the postoperative treatment and follow-up. The histological result G2, pTa represents a bladder tumour with a low grade of malignancy.

In case of $\mathrm{CH}$, histology offers a $100 \%$ correct diagnosis, revealing the adventitia, laminated membrane and the germinal inner layer of the hydatid cyst. Parasitological examination of the cyst content after centrifuging it, shows the presence of protoscolices in the hydatid sand.

\section{Conclusions}

We described an infrequent case of co-existing low grade malignant bladder tumour and a solitary retrovesical secondary $\mathrm{CH}$ in a 55 years old male patient. Despite an efficient surgical treatment for a solitary spleen hydatid cyst 15 years earlier, the patient developed complication by recurrence of $\mathrm{CH}$ in the pelvic area. If a correct postsurgical specific pharmacological treatment had been applied, complete healing would have been insured. In case of this patient, the secondary pelvic cyst remained silent and was only discovered accidentally when the co-existing bladder tumour became symptomatic. CT scan was extremely helpful in diagnosis revealing the presence of daughter cysts inside the large formation. The management of this case was individualized based on localization and size of the cyst and the presence of co-morbidities.

As far as we know there are very scarce descriptions of similar cases in the literature, therefore our case-presentation can be helpful in everyday clinical practice. 


\section{References}

1. Cox, F.E.G.: History of human parasitology. Clin Microbiol Rev 15, 595-612 (2002).

2. Pukar, M.M., Pukar Shabari, M.: Giant solitary cyst of spleen. Int J of Surgery Case Rep 4, 435-437 (2013).

3. Cheung, G., Sahai, A., Billia, M., Dasgupta, P., Khan, M.S.: Recent advances in the diagnosis and treatment of bladder cancer. BMC Med 11, 13. Published online 17 January, 2013 (2013).

4. Kakehi, Y., Hirao, Y., Kim, W.J., Ozono, S., Masumori, N., Miyanaga, N., Nasu, Y., Yokomizo, A.: Bladder cancer working group report. Jpn J Clin Oncol 40 Suppl 1:57-64 (2010).

5. Karaoglu, I., van der Heijden, A.G., Witjes, J.A.: The role of urine markers, white light cystoscopy and fluorescence cystoscopy in recurrence, progression and follow-up of non-muscle invasive bladder cancer. World J Urol 32, 651-659 (2014).

6. Burger, M., Catto, J.W., Dalbagni, G., Grossman, H.B., Herr, H., Karakiewicz, P., Kassouff, W., Kiemeneyg, L.A., La Vecchiah, C., Shariati, S., Lotanj, Y.: Epidemiology and risk factors of urothelial bladder cancer. European Urology 63, 234-241 (2013).

7. Subramaniam, B., Abrol, N., Kumar, R.: Laparoscopic Palanivelu-hydatid-system aided management of retrovesical hydatid cyst. Indian J Urol 29, 59-60 (2013).

8. Angulo, J.C., Sanchez-Chapado, M., Diego, A., Escribano, J., Tamayo, J.C., Martin, L.: Renal echinococcosis: Clinical study of 34 cases. J Urol 157, 787-794 (1997).

9. http://www.who.int/neglected diseases/diseases/en/

10. Saadeh R., Mohamed A.: Urinary retention: Unusual presentation of hydatid cyst. Case report and literature review. The Internet Journal of Surgery 27, 2 (2010).

11. Srinivasan, S.: Retrovesical hydatid cyst presenting with urinary retention and left kidney atrophy. Urol Ann 6, 70-71 (2014).

12. Palanivelu, C., Senthilkumar, R., Jani, K., Rajan, P.S., Sendhilkumar, K., Parthrasarti, R., Rajapandian, S.: Palanivelu hydatid system for safe and efficacious laparoscopic management of hepatic hydatid disease. Surg Endosc 20, 1909-1913 (2006). 